... Orsola Vettori, Direktorin des Spitals Zollikerberg bei Zürich

«Das Spital ist mein Gestaltungsraum»

Daniel Lüthi

Text und Bilder

danielluethi@gmx.ch
Im Eingangsbereich des Spitals fällt der Blick auf eine Steintafel mit den eingemeisselten Worten: «Herr, lass deine Augen offen stehen über dieses Haus.» Orsola Vettori trägt, prominent präsentiert, als Anhänger ein silbernes Kreuz. Gibt es da Parallelen? Hat ihre Anstellung hier religiöse Hintergründe? «Nein», sagt die Direktorin. «Was mich überzeugte, war die Werthaltung, die hinter dem Leitbild der Stiftung steht, die dieses Spital trägt: das Bestreben, den Menschen in seiner Gesamtheit wahrzunehmen.» Und das Kreuz im Ausschnitt? «Das ist das Kreuz, das meine Mutter trug und mich an sie erinnert. Sie starb zu früh.»

\section{Starke Frauen}

Wichtig ist Orsola Vettori der emanzipatorische Hintergrund «ihres» Spitals, das 1998 aus einer Fusion des Diakoniewerkes Neumünster mit der Schweizerischen Pflegerinnenschule entstand. Frauen - Ärztinnen und Krankenschwestern - nahmen mit der «Pflegi» ihr Geschick in die Hand und gründeten ein eigenes Spital. Vettori selbst wuchs zusammen mit drei Schwestern auf - «mein Leben war von Kindheit an von Frauen geprägt», sagt sie heute dazu. «Als in der Schweiz das Frauenstimmrecht eingeführt wurde, war ich 13-jährig. Frauen, die mitgestalten,

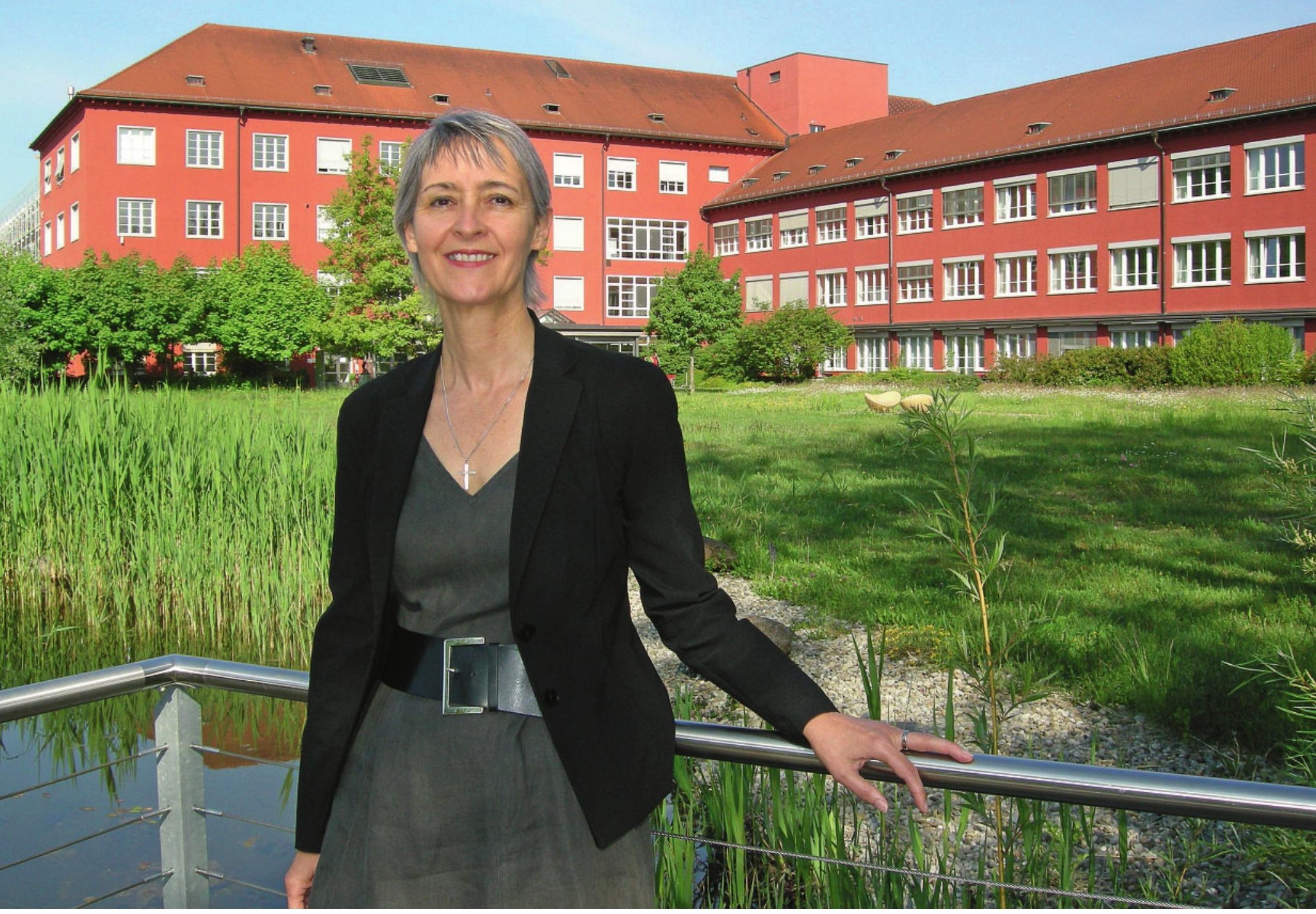


haben mich immer fasziniert - sie begegneten mir wieder, als ich in die Wirtschaft einstieg. Motiviert durch engagierte Mitarbeiterinnen, bemühte sich auch das Planungs- und Architekturbüro, in dem ich 1986 bis 1995 arbeitete, aktiv um Chancengleichheit.»

\section{Unkonventionelles Führungsmodell}

Die Jahre im Planungsbüro, wo interdisziplinäre Zusammenarbeit selbstverständlich war, haben ihre Spuren hinterlassen. So setzt auch das Führungsmodell, das Orsola Vettori im Spital Zollikerberg zu Beginn des vergangenen Jahres eingeführt hat, auf das Miteinander: «Als Erste im Kanton Zürich haben wir in allen unseren Kliniken eine medizinisch-pflegerische Co-Leitung eingeführt.» Konkret heisst das: Das Budget, die Ziele der Klinik und ihre weitere Entwicklung, «die Optimierung der Prozesse», wie es die

\section{«Ich muss Menschen in ihrer Unterschiedlichkeit} gern haben.»

Direktorin nennt - all dies definieren die medizinische und die pflegerische Leitung jeweils gemeinsam, «immer mit dem Blick über beides und aufs Ganze. Früher wollten die Ärzte so und die Pflegenden anders, und mir blieb dann jeweils die Rolle der Schiedsrichterin. Heute wird ein Thema ausbalanciert, bevor es zu mir kommt.»

Die Bilanz nach einem Jahr ist positiv: «Das Modell hat sich ganz klar bewährt. Es ist eine Freude, auf diese Art im Team zu arbeiten.» Womit sie mit «Team» nicht eine Gruppe Gleichgesinnter meint, sondern ein Kollektiv von unterschiedlichen Menschen mit unterschiedlichen Berufen - und unterschiedlichen Stärken.

Eines aber ist auch klar: «Wir praktizieren hier kein generelles Co-Leitungsmodell; in der Unternehmensführung ist diese Organisations-Form nicht anwendbar.» Die zehnköpfige Spitalleitung ziehe sie in alle wichtigen Entscheide mit ein, ergänzt die Direktorin, aber gegenüber der Stiftung für die Betriebsführung verantwortlich sei allein sie.

\section{Vertrauen und Vernetzung}

Was heisst denn «führen» für eine Frau, die sagt, dass sie das besonders gerne macht? «Erstens: Ich muss Menschen in ihrer Unterschiedlichkeit gern haben. Ich muss eine Vorstellung haben, wohin es geht mit diesem Unternehmen, muss seine Gesamtperspektive einnehmen. Und ich muss diese Vorstellung vermitteln können. Dafür braucht es Vertrauen. Und nicht Grabenkämpfe, in denen wir unsere Energie verlieren.» Zusammengefasst: «Das Spital ist mein Gestaltungsraum.»

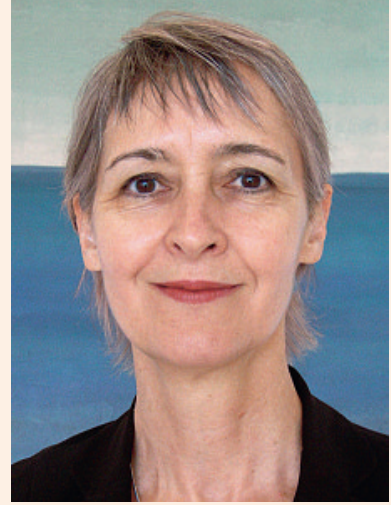

\section{Orsola Vettori}

Dr. iur. Orsola Vettori wurde 1958 in Muri AG geboren. Sie studierte Rechtswissenschaften in Bern und machte dort 1985 auch die Anwaltsprüfung. 1986 bis 1995 arbeitete Vettori in Brugg bei einer Beratungsfirma in Architektur-, Planungs- und Umweltfragen. In dieser Zeit absolvierte sie ein Nachdiplomstudium an der Columbia University in New York und schrieb an der Universität Zürich ihre Dissertation. Anschliessend war sie Stabschefin im Gesundheitsund Umweltdepartement der Stadt Zürich. In Glasgow GB machte sie den Master oft Business Administration. Seit 2001 ist Orsola Vettori eine italienisch-schweizerische Doppelbürgerin - Direktorin des Spitals Zollikerberg, das der Stiftung «Diakoniewerk Neumünster - Schweizerische Pflegerinnenschule» gehört. Es beschäftigt rund 800 Mitarbeitende, 80 Prozent davon sind Frauen. Das Spital hat 200 Betten und macht jährlich einen Umsatz von 100 Millionen Franken. Orsola Vettori ist mit dem Dermatologen und Musiker Jochen Wenz verheiratet, der in Mannheim/Deutschland lebt. Sie selbst wohnt in der Stadt Zürich.

Dies sind die Massstäbe, die die Direktorin bei sich selbst ansetzt: «Ich muss integrieren können. Ich muss eine gute Innen- und eine gute Aussenministerin sein, das heisst gut vernetzen können, intern und mit anderen Spitälern oder mit Behörden. Ich muss gut kommunizieren - und gut rekrutieren können.»

Bisweilen erinnert das, was sie sagt, und die Art, wie sie es sagt, an die Rede einer Politikerin. Orsola Vettori wehrt sich, vehement, mit offenen Augen und klaren Sätzen: «Ich bin keine Gesundheitspolitikerin. 
Mein Fokus ist das Spital. Aus dieser Perspektive bringe ich mich im Gesundheitswesen ein.»

\section{Qualität für alle}

Ein Spital muss heute neben medizinischer und pflegerischer Qualität auch andere Ansprüche abdecken. Patienten wollen sich wohlfühlen, wünschen gutes Essen, ein schönes Zimmer, Privatsphäre. «Ja, die Erwartungen sind gestiegen. Vor ein paar Jahren noch galt ein Viererzimmer als normal, heute bieten wir nur noch Einzel- und Zweierzimmer an.» Mehr Luxus - höhere Kosten? «Die Investitionskosten machen bloss etwas über zehn Prozent der Gesamtkosten aus. Der grösste Kostenblock im Spital sind die Personalkosten. Heute arbeiten rund

\section{«Wir halten uns im Markt, indem wir versuchen, neue Wege zu gehen und den Spagat zwischen hoher Qualität und Kostenbewusstsein zu meistern.»}

100 Ärztinnen und Ärzte im Spital Zollikerberg, 2003 waren es weniger als 70. Damals galt Teilzeitarbeit im ärztlichen Bereich noch als unmöglich, und die Arbeitszeit betrug oft mehr als 70 Wochenstunden. Heute gilt bis auf Oberarztstufe die 50-StundenWoche. Bei uns gibt es sogar einen Chefarzt, der Teilzeit arbeitet.»

Und wie sieht die angesprochene Arbeits-FreizeitBalance bei Orsola Vettori selbst aus? «Ich wohne und arbeite hier in Zürich, mein Mann lebt in Deutschland. Deshalb sind bei mir die Wochenenden in aller Regel heilig, damit wir die Zweisamkeit geniessen können. Ich freue mich jedes Mal wie ein Maikäfer, ihn zu sehen, seit 17 Jahren. Unser Modell ermöglicht mir, während der Woche engagiert und zeitintensiv zu arbeiten.»

\section{Wirtschaftlichkeit ...}

«Was mich interessiert, ist die Frage: Wie führe ich dieses Spital erfolgreich in die Zukunft?», sagt Orsola Vettori. Ein Blick aus dem Fenster ihres Direktionsbüros liefert Hinweise, was das konkret heissen könnte: Der neue Westflügel, der einen Gebäudeteil aus den dreissiger Jahren ersetzt, ist vollständig eingerüstet, mehrere Krane ragen in den Himmel. «Eine zukunftsweisende Idee wird hier verwirklicht», kommentiert Vettori. Der Neubau, der entsteht, wird ab kommendem Jahr eine Zweigstelle der in Zurzach domizilierten «RehaClinic» beherbergen, mit der das
Spital ein Kooperationsabkommen abgeschlossen hat. «Damit können wir unseren Patienten eine ganze Prozesskette anbieten. Eine stationäre Reha-Klinik in einer grossen Agglomeration gibt es in der Schweiz bisher noch nicht.» Ein wenig Stolz schwingt da unüberhörbar mit.

«Wir halten uns im Markt, indem wir versuchen, neue Wege zu gehen und den Spagat zwischen hoher Qualität und Kostenbewusstsein zu meistern. Die zusatzversicherten Patienten, die wir uns erhalten wollen, haben berechtigterweise hohe Ansprüche. Unsere Anstrengungen um die Qualität kommen aber - anders als in den Privatkliniken - allen Patienten, auch den Allgemeinversicherten, zugute.»

Mit dem neuen System der diagnosebezogenen Fallgruppen (DRG) werde die Wirtschaftlichkeit eine noch grössere Rolle spielen, sagt Vettori. «Wir schauen dieser Entwicklung mit einer gespannten Gelassenheit entgegen.» Droht mit SwissDRG eine Entmenschlichung der Medizin? «Nein, das können wir uns gar nicht leisten, dann käme ganz schnell niemand mehr zu uns.»

Wie auch immer: Effizienz ist hier angesagt, wirtschaftlicher Erfolg. Das zeigt der Blick auf diese Seite der Spitalanlage.

\section{... und Menschlichkeit}

Ein ganz anderes Bild auf der anderen Seite: ein Park mit Magerwiese und Teich, die ein Gefühl von Beschaulichkeit und Naturverbundenheit vermitteln. Beide Seiten gehören zu diesem Spital. Und beide Seiten gehören zu Orsola Vettori: die klare geschäftliche und die weiche menschliche, naturverbundene. Jetzt erinnert sie sich an ihre Jugend, an den grossen Bauernhof im aargauischen Muri, an den Vater, der aus einer Weinbauernfamilie in Norditalien stammte, und an die Mutter und ihren grossen Garten. Es war die Schwarzenbach-Zeit, und Orsola Vettori war damals Italienerin. «Einmal sagte mir ein Schulkamerad: «Morgen gehen wir darüber abstimmen, ob du am Montag noch mit uns zur Schule kommen kannst oder nicht ১.» Zur gleichen Zeit machte die Lehrerin aus «Orsola» wie selbstverständlich «Ursula». Fremd sein im eigenen Land - das kennt Orsola Vettori aus jener Zeit. Heute sagt sie als Seconda: «Ich habe einen starken Bezug zu Italien, es ist mein Vaterland - aber ich fühle mich als Schweizerin.»

Wobei sie die Schweiz als multikulturelles Land sieht, als Ganzes mit vielen verschiedenen Einzelteilen. Und womit sich ein Kreis schliesst - jener zu Orsola Vettoris Verständnis von Team: Vielfalt und die Art, damit umzugehen, sagt sie, sind die Voraussetzungen für Einheit.

\section{Die nächste «Begegnung mit ...»}

Am Ende jeden Monats stellt die Schweizerische Ärztezeitung eine Persönlichkeit vor, die sich im Gesundheitswesen engagiert. Im August schildert Daniel Lüthi seine Begegnung mit Beppe Savary, Allgemeinmediziner und Notarzt im Onsernone-Tal. 